\title{
Biological Analysis of Simvastatin-releasing Chitosan Scaffold as a Cell-free System for Pulp-dentin Regeneration
}

Diana G. Soares, DDS, MS, PhD, * Giovanna Anovazzi, DDS, MS, PhD, ${ }^{\dagger}$

Ester Alves F. Bordini, DDS, MS, PbD, ${ }^{\neq}$Uxua O. Zuta, DDS, MS, ${ }^{\neq}$

Maria Luísa A. Silva Leite, DDS, MS, ${ }^{\neq}$Fernanda G. Basso, DDS, MS, PbD, ${ }^{\ddagger}$

Josimeri Hebling, DDS, MS, PbD, ${ }^{\dagger}$ and Carlos A. de Souza Costa, DDS, MS, PbD

\section{Ahstract}

Introduction: The improvement of biomaterials capable of driving the regeneration of the pulp-dentin complex mediated by resident cells is the goal of regenerative dentistry. In the present investigation, a chitosan scaffold (CHSC) that released bioactive concentrations of simvastatin (SIM) was tested, aimed at the development of a cell-free tissue engineering system. Methods: First, we performed a dose-response assay to select the bioactive dose of SIM capable of inducing an odontoblastic phenotype in dental pulp cells (DPCs); after which we evaluated the synergistic effect of this dosage with the CHSC/DPC construct. SIM at $1.0 \mu \mathrm{mol} / \mathrm{L}$ (CHSCSIM1.0) and $0.5 \mu \mathrm{mol} / \mathrm{L}$ were incorporated into the CHSC, and cell viability, adhesion, and calcium deposition were evaluated. Finally, we assessed the biomaterials in an artificial pulp chamber/3-dimensional culture model to simulate the cell-free approach in vitro. Results: SIM at $0.1 \mu \mathrm{mol} / \mathrm{L}$ was selected as the bioactive dose. This drug was capable of strongly inducing an odontoblastic phenotype on the DPC/ CHSC construct. The incorporation of SIM into CHSC had no deleterious effect on cell viability and adhesion to the scaffold structure. CHSC-SIM1.0 led to significantly higher calcium-rich matrix deposition on scaffold/dentin disc assay compared with the control (CHSC). This biomaterial induced the migration of DPCs from a 3-dimensional culture to its surface as well as stimulated significantly higher expressions of alkaline phosphatase, collagen type 1 alpha 1, dentin matrix acidic phosphoprotein 1, and dentin sialophosphoprotein on 3-dimensional-cultured DPCs than on those in contact with CHSC. Conclusions: CHSCSIM1.0 scaffold was capable of increasing the chemotaxis and regenerative potential of DPCs. (J Endod 2018;44:971-976)

\section{Key Words}

Cell differentiation, dental pulp, scaffolds, tissue engineering

W ith the advances in tissue engineering and regenerative dentistry, efforts have been directed to the development of bioactive scaffolds for pulp-dentin complex regeneration. The design of biomaterials in combination with the Significance

This study showed the chemotactic and bioactive potentials of chitosan scaffolds associated with low-dose simvastatin on dental pulp cells. Therefore, this technology may be considered a promising strategy as a cell-free system for pulp-dentin complex regeneration.

controlled release of signaling clues to direct the fate of mesenchymal stem cells (MSCs) into osteo/odontoblastic phenotypes is a highly promising therapeutic strategy to achieve dental and craniofacial mineralized tissue engineering (1-3). To guide adequate tissue regeneration, desirable aspects of scaffold chemistry should be addressed, including the creation of a polymeric network that mimics extracellular matrix components, allowing cell interaction to occur (4).

Porous chitosan scaffolds (CHSCs), which possess polymeric chains structurally similar to glycosaminoglycan (GAG), have been reported to be a suitable substrate for dental pulp cell (DPC) adhesion, proliferation, and odontoblastic differentiation upon adequate cell signaling (5). Chitosan has generated great interest in the tissue engineering field because of its good biocompatibility, biodegradability into nontoxic components, protein affinity, and hemostatic and antimicrobial potential $(6,7)$. Therefore, CHSCs might be useful in direct pulp capping therapy because they may provide a biocompatible matrix for the adhesion, proliferation, and differentiation of endogenous DPCs into odontoblastlike cells capable of depositing and mineralizing dentin matrix, thus restoring dentin integrity at the pulp-dentin border.

The release of bioactive substances for a direct influence on the behavior of ingrowing cells on the scaffold structure is considered a hallmark of tissue engineering applications, thus increasing the amount and quality of neotissue genesis in a shorter time (4). Researchers have found that local application of statins is capable of strongly accelerating the repair of bone defects in vivo $(8,9)$. Recent review articles regarding this specific topic concluded that local delivery of simvastatin (SIM) from biomaterials seems to be more reliable than systemic administration for bone regeneration; however,

From the *Department of Operative Dentistry, Endondontics and Dental Materials, Bauru School of Dentistry, University of São Paulo-USP; Departments of ${ }^{\ddagger}$ Physiology and Pathology, and ${ }^{\dagger}$ Orthodontics and Pediatric Dentistry, Araraquara School of Dentistry, Universidade Estadual Paulista-UNESP, São Paulo, Brazil.

Address requests for reprints to Dr Carlos A. de Souza Costa, Department of Physiology and Pathology, Araraquara School of Dentistry, UNESP, Humaitá Street, 1680 Centro, 14801-903, Araraquara, SP, Brazil. E-mail address: casouzac@foar.unesp.br

0099-2399/\$ - see front matter

Copyright (C) 2018 American Association of Endodontists.

https://doi.org/10.1016/j.joen.2018.02.014 
depending on the released dosage, SIM can either accelerate or retard mineralized neotissue genesis $(10,11)$. At low concentrations, these drugs feature pleiotropic effects with mesenchymal stem cells, increasing the expression of several osteo/odontoblastic markers, such as dentin sialophosphoprotein (DSPP), dentin matrix acidic phosphoprotein 1 (DMP-1), alkaline phosphatase (ALP), collagen type 1 alpha 1 (Col1A1), osteocalcin, osteopontin, runt-related transcription factor 2 , and bone morphogenetic protein 2 (BMP-2), leading to intense mineralized matrix deposition in vitro (12-15). Accordingly, the objective of this study was to develop a porous chitosan scaffold capable of releasing SIM at a bioactive level, stimulating the migration and odontoblastic phenotype expression of DPCs, aimed at the development of a cell-free tissue engineering system for regeneration of the pulp-dentin complex. The tested hypothesis was that a chitosan scaffold associated with a low dose of simvastatin can induce DPC migration and odontoblastic differentiation.

\section{Establishment of DPCS \\ Materials and Methods}

Primary culture of DPCs was obtained by enzymatic dissociation of fresh pulp tissue from sound human third molars from a 24-year-old male patient as described in detail by Soares et al (5). The patient provided informed consent according to the Declaration of Helsinki (protocol \#30939314.5.0000.5416), and the study was approved by the Research Ethics Committee of the Araraquara School of Dentistry, Universidade Estadual Paulista, São Paulo, Brazil. The cells were cultured in complete alpha minimum essential medium ( $\alpha$-MEM; Invitrogen, Carlsbad, CA) supplemented with $10 \%$ fetal bovine serum, L-glutamine, and $1 \%$ penicillin-streptomycin and used from passages 3 to 6 for the experimental protocols.

\section{Selecting Bioactive Dosages of Simvastatin}

The DPCs were seeded on 96-well plates (Corning, Corning, NY) $\left(1 \times 10^{4}\right.$ cells/well $)$ in complete $\alpha$-MEM and incubated for 24 hours. They were then cultured in $100 \mu \mathrm{L}$ osteogenic medium (complete $\alpha$-MEM plus $50 \mu \mathrm{g} / \mathrm{mL}$ ascorbic acid and $5 \mathrm{mmol} / \mathrm{L} \beta$-glycerophosphate; Sigma-Aldrich, St Louis, M0) supplemented with $0 \mu \mathrm{mol} / \mathrm{L}$, $0.01 \mu \mathrm{mol} / \mathrm{L}, 0.1 \mu \mathrm{mol} / \mathrm{L}$, or $1 \mu \mathrm{mol} / \mathrm{L}$ SIM (Sigma-Aldrich) for up to 21 days. The culture medium supplemented or not with SIM was replaced every 48 hours. The metabolic activity of cells (alamarBlue Assay; Thermo Fisher Scientific, Waltham, MA), ALP activity (thymolphthalein monophosphate substrate-based assay), calcium deposition (alizarin red), and cell migration (wound healing and transwell assays) were evaluated $(5,16)$. Detailed information can be found in the Supplementary Material.

\section{Synergistic Potential of SIM and CHSEs}

A $2 \%$ chitosan solution $(75 \%-85 \%$ deacetylated, mol wt $310,000-375,000 \mathrm{~d}$; Sigma-Aldrich) in 2\% aqueous solution of glacial acetic acid (Sigma-Aldrich) was poured $(500 \mu \mathrm{L})$ into polystyrene tubes, frozen at $-80^{\circ} \mathrm{C}$ for 4 hours, and freeze-dried at $-56 \mathrm{C}$ overnight to obtain CHSCs. The surface and transversal slices of CHSCs were evaluated using scanning electron microscopy (12-15 kV [JMS6610V Scanning Microscope; JEOL, Tokyo, Japan]). The overall porosity was calculated by Image software (National Institutes of Health, Bethesda, MD) in 3 samples at $100 \times$ magnification $(n=3)$, and the CHSC featured a mean porosity of $35.1 \% \pm 1.9 \%$.

CHSC samples (5-mm diameter and 1-mm thickness) were sterilized in $70 \%$ ethanol (30 minutes) under a vacuum, washed in phosphate-buffered saline (PBS) (3 times of 30 minutes), and incubated in complete $\alpha$-MEM overnight at $37^{\circ} \mathrm{C}$ and $5 \% \mathrm{CO}_{2}$. The cells $\left(1 \times 10^{5}\right.$ cells $)$ in $3 \mu \mathrm{L} \alpha$-MEM were seeded onto the scaffolds followed by incubation for 30 minutes to allow cells to adhere exclusively to the scaffold structure and cultivation in $500 \mu \mathrm{L} \alpha$-MEM for 24 hours. Thereafter, the DPC/scaffold constructs were cultivated on osteogenic medium supplemented or not with $0.1 \mu \mathrm{mol} / \mathrm{L}$ SIM for up to 21 days. The following groups were established: CHSC/DPC construct cultivated with osteogenic medium and CHSC + SIM/DPC construct cultivated with osteogenic medium supplemented with $0.1 \mu \mathrm{mol} / \mathrm{L}$ SIM. Cell viability (Live/Dead Assay, Invitrogen), proliferation (alamarBlue Assay), cell spread (F-actin staining), ALP activity (p-nitrophenyl phosphate substrate), mineralized matrix deposition (alizarin red), and cell migration (transwell) were evaluated at different time points $(5,16)$.

\section{Bioactive Potential of SIM-releasing GHSCs}

The CHSC was immersed in PBS ( $\mathrm{pH}=7.4$ ) with $15 \%$ dimethyl sulfoxide (Sigma-Aldrich) with or without $1 \mathrm{mmol} / \mathrm{L}$ SIM for 24 hours at $37^{\circ} \mathrm{C}$ in order to calculate the percentage of drug release. After the determination of $10 \%$ SIM release within 24 hours, $1.0 \mu \mathrm{mol} / \mathrm{L}$ (SIM1.0) and $0.5 \mu \mathrm{mol} / \mathrm{L}$ (SIM0.5) SIM were incorporated into CHSCs, aimed at the release of $0.1-0.05 \mu \mathrm{mol} / \mathrm{L}$ SIM. The cell viability (Live/ Dead assay), spread (F-actin staining), and calcium-rich mineral deposition (alizarin red) were evaluated on DPCs seeded on CHSC, CHSCSIM1.0, and CHSC-SIM0.5 adapted to circular perforations (1-mm thick and 4-mm diameter) prepared on human dentin discs (2-mm thick and 8-mm diameter, protocol \#30939314.5.0000.5416) (17).

To evaluate the potential of the biomaterials as a cell-free approach for dentin regeneration, we prepared an in vitro model of an artificial pulp chamber (APC) with 3-dimensional (3D)-cultured DPCs. Human dentin discs (1-mm thick and 8-mm diameter) containing a central perforation (1-mm thick and 4-mm diameter) were adapted to the APC between 2 silicon 0-rings (5). The 3D matrix, composed of 1:1 Hydromatrix (Sigma-Aldrich) and type 1 collagen (rat tail, $3.67 \mathrm{mg} / \mathrm{mL}$; Corning), was pipetted onto the pulpal sides of dentin discs $(100 \mu \mathrm{L})$. After $\mathrm{pH}$ neutralization, $1 \times 10^{5}$ DPCs were seeded on the 3D matrix, and 24 hours thereafter the scaffold (5-mm diameter and 1-mm thick) was adapted to the dentin discs in intimate contact with the 3D cell culture. The set was incubated in complete $\alpha$-MEM (with no osteogenic supplementation) for 21 days, and the presence of viable cells on the 3D matrix and scaffold surfaces was evaluated by the Live/ Dead assay (5). The gene expression of ALP, Col1A1, DSPP, and DMP-1 was evaluated in the 3D culture by real-time polymerase chain reaction at the 21-day period (5). Detailed information can be found in the Supplementary Material.

\section{Statistical Analysis}

The experiments were performed twice to ensure reproducibility. Data were compiled and normalized by a negative control group (no SIM supplementation). Data were then analyzed using Student $t$ tests and 1- or 2-way analysis of variance complemented by the Tukey test for observation of the significant differences between the study groups. A value of $P<.05$ was considered to be statistically significant.

\section{Results}

\section{Bioactive Potential of Low-dose SIM with DPES}

Regarding cell viability, no significant difference was observed among groups on 1 day; however, a significant reduction in the proliferative capability was observed for the cells cultivated in contact with SIM at 7 and 14 days in comparison with the negative control (Fig. 1A). The dose response for SIM inducing odontoblastic phenotypes on DPCs showed that $0.1 \mu \mathrm{mol} / \mathrm{L}$ SIM featured a bioactive 

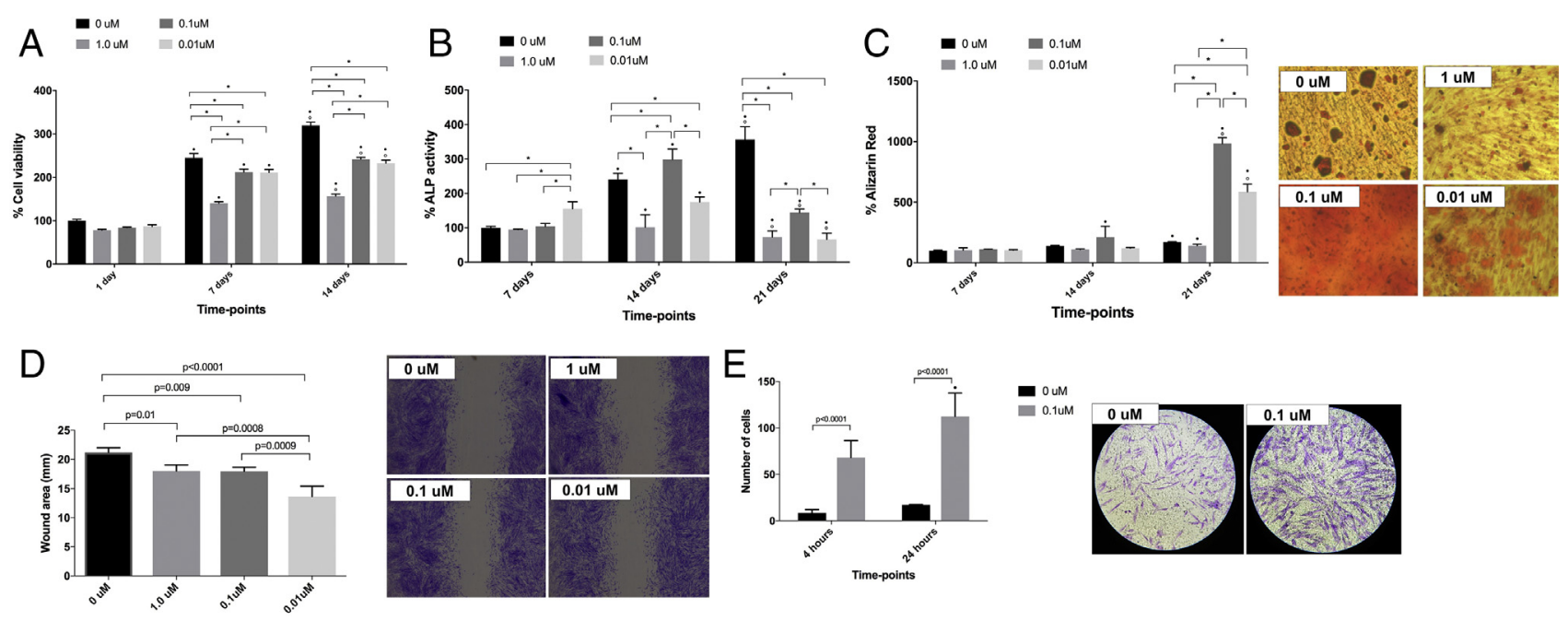

Figure 1. SIM dose response. $(A)$ alamarBlue, $(B)$ ALP activity, and $(C)$ alizarin red assays for the SIM dose response. $\bullet$, a significant difference at 1 day for each group. ${ }^{\circ}$, a significant difference at 7 days for each group. — , differences between groups at each time point (2-way analysis of variance/Tukey test, $n=6, \alpha=5 \%$, $* P<.0001)$. Note the representative images of mineralized nodule deposition at 21 days. $(D)$ Wound healing assay. - , differences between groups $(1$-way analysis of variance/Tukey test, $n=4, \alpha=5 \%)$. Note the representative images of the wound area stained with crystal violet at 24 hours. $(E)$ Transwell assay. - , differences between groups. $\bullet$, a significant difference between time points for each group (Student $t$ test; $n=4, \alpha=5 \%$ ). Note the representative images of migrating cells stained with crystal violet at 24 hours.

potential, significantly increasing ALP activity at 14 days and mineralized matrix deposition at 21 days in comparison with the control (Fig. $1 B$ and $C$ ). All tested SIM concentrations featured chemotactic potential, as shown by a significant reduction of the wound area relative to the control (Fig. 1D). The transwell assay demonstrated that $0.1 \mu \mathrm{mol} / \mathrm{L}$ SIM, selected as the bioactive dose, showed an intense capability of inducing active cell migration through transwell membranes at both 4 and 24 hours (Fig. $1 E$ ).

\section{Low-dose SIM Featuring a Synergistic Effect with CHSEs}

The CHSC featured homogeneous porous architecture at the material surface and in transverse slices (Fig. $2 A$ and $B$ ). The cells were capable of proliferating significantly on the scaffold structure regardless of the presence of SIM in the culture medium (Fig. 2C). The fluorescence assays showed that cells remained viable on scaffolds and were organized into clusters inside their porous structures (Fig. $2 D$ and $E)$. The cells cultivated in contact with $0.1 \mu \mathrm{mol} / \mathrm{L}$ SIM featured increased ALP activity and mineralized matrix deposition in comparison with the control (Fig. $2 F$ and $G$ ). Finally, the transwell assay showed that the DPCs migrated actively in the presence of CHSCs compared with the control. This phenomenon was more intense when the culture medium was supplemented with SIM, corroborating the previous analysis (Fig. 2H).

\section{Bioactive Potential of SIM-releasing CHSC with DPGS}

Figures $3 A$ and $4 A$ show the experimental design for dentin disc and APC/disc set assays, respectively. Both SIM concentrations allowed DPCs to adhere to the CHSC structure, remaining viable (Fig. $3 B$ and $C$ ). Intensely stained actin fibers were observed in the cells seeded on CHSCSIM1.0 (Fig. 3C). The cells seeded on CHSC-SIM1.0 and CHSC-SIM0.5 deposited an increased amount of mineralized matrix in comparison with the control (CHSC), with calcium-rich matrix being observed on dentin discs; however, a significant difference from the CHSC group was observed only for the CHSC-SIM1.0 group (Fig. $3 D$ and $E$ ). The APC/disc set assay showed that the materials had no cytotoxic effect on 3D-cultured DPCs (Fig. 4B), with some cells being capable of migrating and adhering to SIM-containing scaffolds after the 21-day incubation time (Fig. 4C). CHSC-SIM1.0 significantly increased the gene expression of ALP, Col1, DSPP, and DMP-1 in comparison with the control (Fig. $4 D-G$ ).

\section{Discussion}

In this study, we hypothesized that SIM-releasing CHSCs could be an interesting candidate for a cell-free tissue engineering system for pulp-dentin regeneration. To that end, we performed a series of experiments to develop a porous CHSC capable of releasing bioactive concentrations of SIM to induce DPC migration, adhesion, spread, and proliferation as well as the expression of odontoblastic phenotypes; $0.1 \mu \mathrm{mol} / \mathrm{L}$ SIM featured an intense bioactive potential because it intensely increased ALP activity, deposition of mineralized matrix, and DPC migration in both culture plate and scaffold assays. When the DPC/scaffold construct was cultured in $0.1 \mu \mathrm{mol} / \mathrm{L}$ SIMsupplemented medium, the cells were able to migrate and proliferate inside the CHSC porous matrix, remaining viable. Previous studies have shown that $0.01-1 \mu \mathrm{mol} / \mathrm{L}$ SIM has a stimulatory effect on osteoblastic/odontoblastic differentiation of mesenchymal stem cells in vitro and in vivo, involving phosphorylation of extracellular signal-regulated protein kinases 1 and 2, in a mechanism that seems to be independent of the mevalonate pathway (18-22). Liu et al (8) also found that SIM at concentrations of 0.1 and $0.2 \mu \mathrm{mol} / \mathrm{L}$ significantly increased bone marrow stem cell (BMSCs) migration by $30.7 \%$ and $36.0 \%$ after 24-hour incubation. In vivo assays showed that local application of SIM led to the recruitment of autogenous BMSCs to injured areas $(6,23)$.

CHSCs were then incubated with $0.5 \mu \mathrm{mol} / \mathrm{L}$ and $1.0 \mu \mathrm{mol} / \mathrm{L} \mathrm{SIM}$ for drug surface binding (24), thus obtaining scaffolds capable of releasing $0.05-0.1 \mu \mathrm{mol} / \mathrm{L}$ SIM because we detected that this method resulted in $10 \%$ SIM release in the first 24 hours. This low release was attributed to the lipophilicity of SIM, resulting in its progressive dissolution and diffusion from scaffold surfaces in contact with PBS $(25,26)$. Cells were seeded on scaffold surfaces adapted to dentin discs, and we observed that the incorporation of SIM into the CHSC had no influence 
A
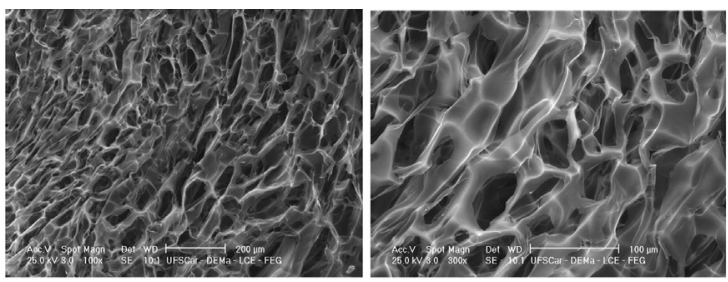

$\mathrm{B}$
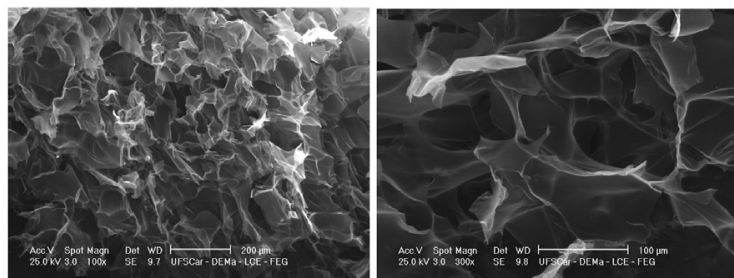

C

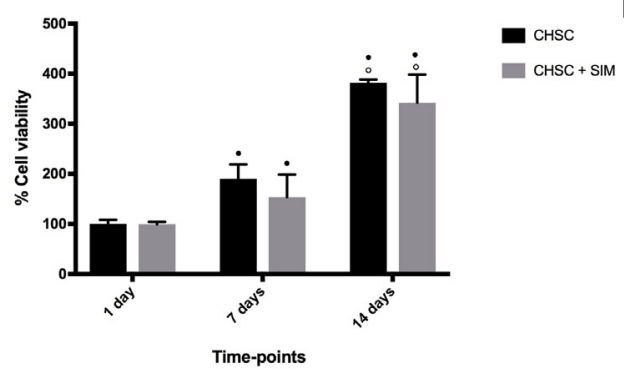

$\mathrm{F}$

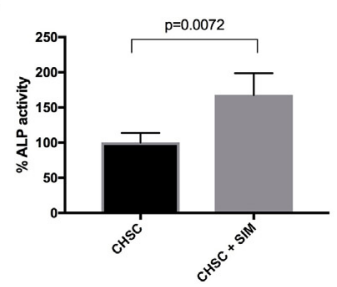

G

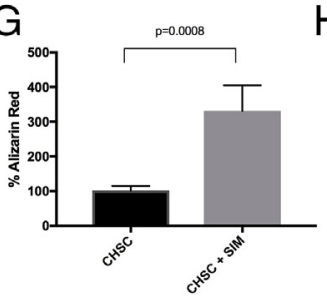

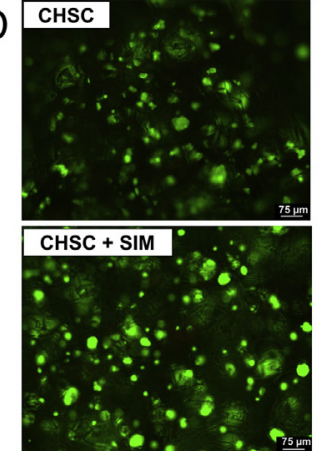

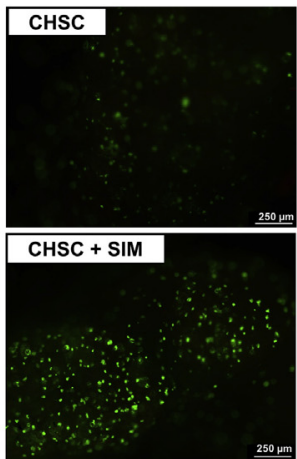

$\mathrm{E}$

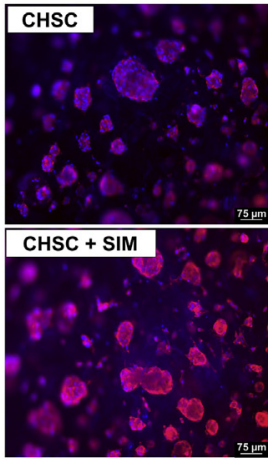

$\mathrm{H}$
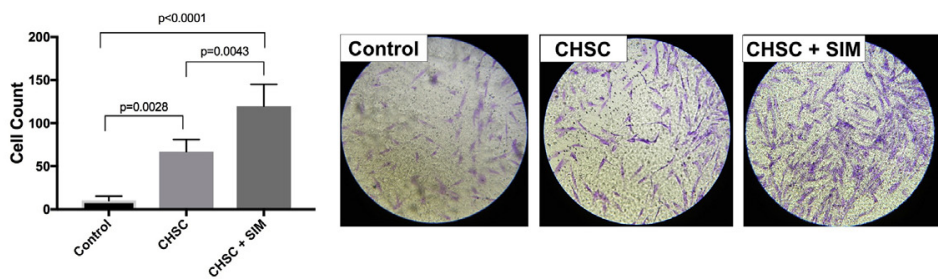

Figure 2. The synergistic potential of SIM and CHSC. $(A$ and $B)$ Scanning electron microscopic images of the CHSC surface and transverse slices $(100 \times$ and $300 \times$ ), respectively. Note the homogeneous porous structure. $(C)$ alamarBlue assay. No significant differences between groups were observed (Student $t$ test, $n=6, \alpha=5 \%)$. $\bullet$ a significant difference at 1 day for each group. ${ }^{\circ}$, a significant difference at 7 days for each group (2-way analysis of variance/Tukey test, $n=6 ; \alpha=5 \%)$. (D) Representative images of the Live/Dead assay for each group at the (left) material surface and (right) transverse slice (right). Green fluorescence indicates viable cells. Red fluorescence indicates dead cells. $(E) \mathrm{F}$-actin staining at the material surface for each material. Red fluorescence indicates actin fibers. Blue fluorescence indicates nuclei. $(F)$ ALP activity and $(G)$ alizarin red assays. - , differences between groups (Student $t$ test, $n=6, \alpha=5 \%$ ). (H) Transwell assay. - , differences between groups (1-way analysis of variance/Tukey test, $n=6, \alpha=5 \%$ ). Note the representative images of migrating cells stained with crystal violet at 24 hours.

on cell viability. Thus, DPCs were capable of migrating inside the scaffold pore structure, with cells of CHSC-SIM1.0 featuring intensely stained actin fibers. After 21 days of dentin/scaffold/DPC construct cultivation in osteogenic medium, we observed that, in the presence of SIM, the cells deposited a high amount of mineralized matrix, which was observed on material structure and at the surfaces
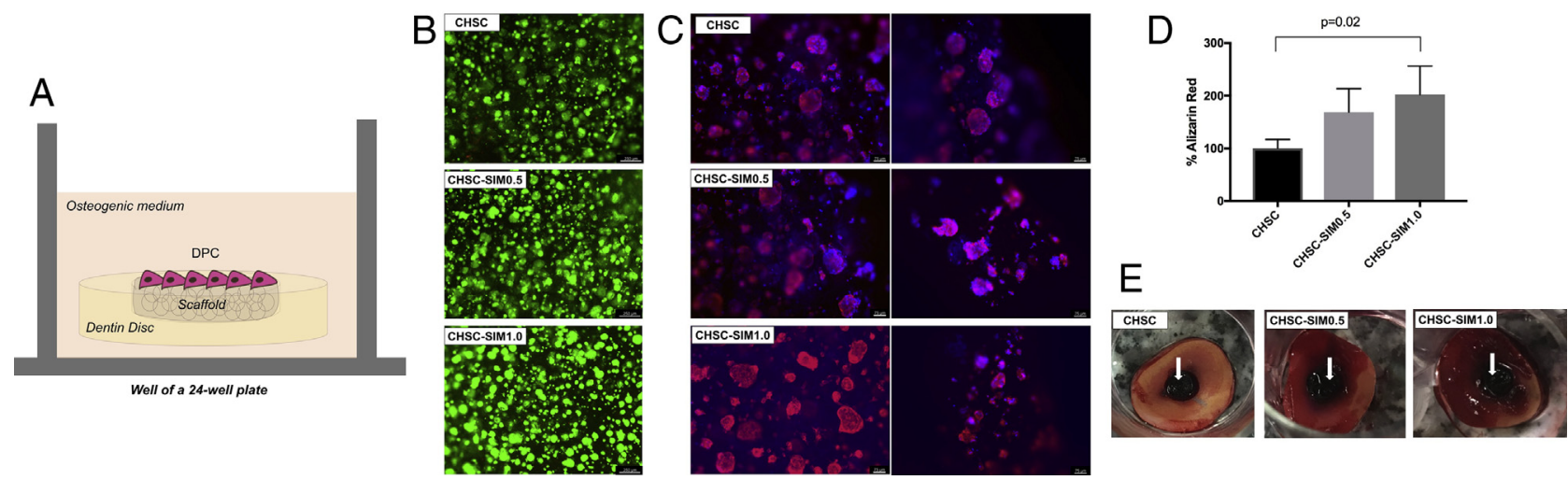

Figure 3. Dentin disc assay. (A) A schematic representation of DPC cultivation on the scaffold adapted to dentin discs. (B) Live/Dead assay. Green fluorescence indicates viable cells. Red fluorescence indicates dead cells. $(C)$ F-actin staining at the material surface and transverse slices, respectively. Red fluorescence indicates actin fibers. Blue fluorescence indicates the nuclei. $(D)$ Alizarin red assay and $(E)$ representative images for each group. The arrows indicate the scaffolds. Red staining represents the calcium-rich matrix. — , differences between groups (1-way analysis of variance/Tukey test, $n=6, \alpha=5 \%$ ). 

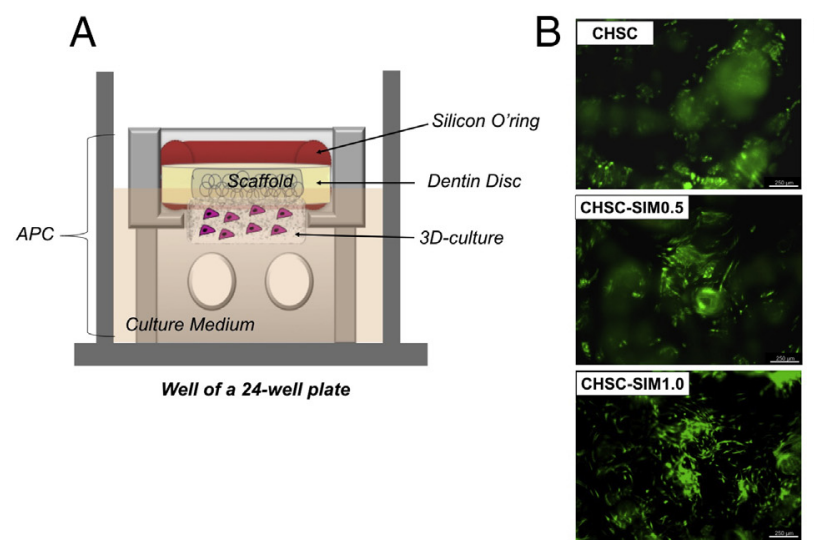
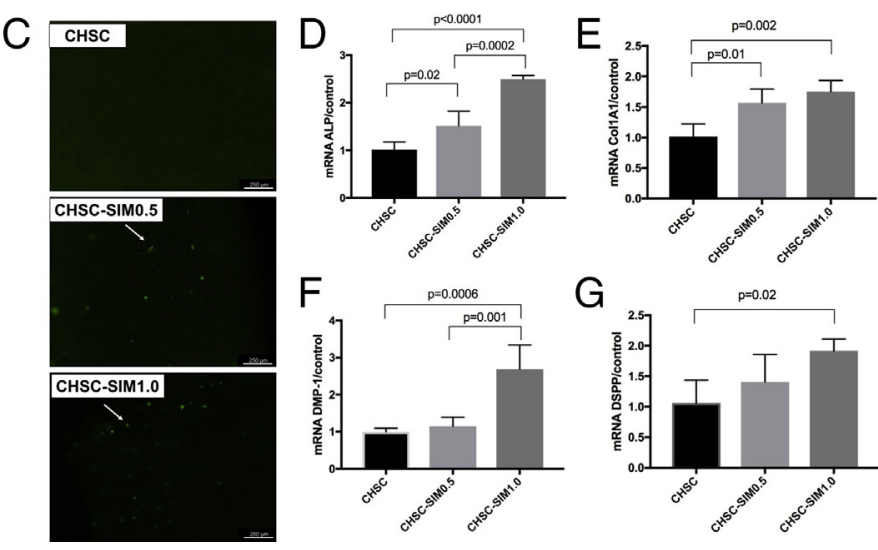

Figure 4. APC assay. (A) A schematic representation of APC/disc set with 3D culture. (B) Live/Dead assay for 3D culture and $(C)$ scaffold surface in contact with 3D culture. Green fluorescence indicates viable cells. Red fluorescence indicates dead cells. The arrows indicate live cells at the scaffold surface. $(D)$ ALP, $(E)$ Col1A1, $(F)$ DMP-1, and $(G)$ DSPP mRNA gene expression. — , differences between groups (1-way analysis of variance/Tukey test, $n=6, \alpha=5 \%$ ).

of dentin discs, especially in the CHSC-SIM1.0 group. Gentile et al (9) showed that chitosan-gelatin scaffolds engrafted with SIM-loaded poly(lactic-co-glycolic acid microspheres released around $1 \mu \mathrm{mol} / \mathrm{L}$ within 24 hours, which was cytocompatible with BMSCs seeded on their surfaces, leading to increased ALP activity and calcium deposition in vitro, such as observed in the present investigation. Using a mouse model of gap fracture, Tai et al (27) showed that the release of simvastatin in the range of $0.02-0.53 \mu \mathrm{g} /$ day from a poly(lactic-co-glycolic acid/hydroxyapatite scaffold enhanced initial callus formation, neovascularization, and cell ingrowth in the grafted bone, indicating that a biomaterial able to release low-dose SIM facilitates bone regeneration in vivo. Several other researchers also reported that SIM-loaded scaffolds in different polymeric matrices increased the osteogenic potential of BMSCs in vitro, leading to ectopic bone formation and bone defect regeneration in vivo, in a mechanism that involves the gene/protein expression of bone markers, such as Col1A1, ALP, BMP-2, bone sialoprotein, osteocalcin, osteopontin, and runt-related transcription factor $2(8,9,24,28-32)$.

Regarding pulp-dentin complex regeneration, few investigations have assessed the effects of SIM-loaded biomaterials. Miyazawa et al (33) developed a gelatin hydrogel polylactic acid micelles loaded with low concentrations of SIM. These authors determined that this biomaterial enhanced ALP activity, calcium deposition, and BMP-2 secretion by DPCs seeded on its structure. After subcutaneous implantation into mice, the constructs containing SIM exhibited increased DSP expression and calcium deposition. In a previous study, Asl Aminabadi et al (34) applied SIM at concentrations of $1 \mu \mathrm{mol} / \mathrm{L}, 5 \mu \mathrm{mol} / \mathrm{L}$, and $10 \mu \mathrm{mol} / \mathrm{L}$, along with highly viscous sodium carboxyl methylcellulose as a carrier, to exposed pulp in human primary molars. Those authors observed that the higher SIM concentrations led to dental pulp inflammation and that a small amount of this substance stimulated dentin bridge formation at the pulp exposure site. SIM at $1 \mu \mathrm{mol} / \mathrm{L}$ induced odontoblastlike cell differentiation from host DPCs followed by deposition of mineralized tissue at the pulp-dentin border; however, pulp inflammation was still detected. These data may be correlated with the toxic effect of SIM at high concentrations, as also observed in the present investigation for $1 \mu \mathrm{mol} / \mathrm{L}$ SIM.

According to the experiments cited previously, regardless of scaffold composition, SIM plays a positive role on mineralized tissue regeneration. Therefore, future experiments should be performed to select the ideal SIM-loaded scaffold for pulp-dentin regeneration. The advantage of chitosan is the similarity of this molecule with GAGs.
When dissolved in acid medium $(\mathrm{pH}<6.0)$, the positively charged amine groups $\left(\mathrm{NH}_{3}{ }^{+}\right)$of chitosan allow the establishment of electrostatic bonding with GAGs from host tissue, further allowing its interaction with the surrounding extracellular matrix with no foreign body reaction $(6,7)$. Chitosan also allows the establishment of cross-linking by $\mathrm{NH}_{3}{ }^{+}$groups. Therefore, the drug release, polymer degradation rate, and mechanical properties can be modulated. Finally, chitosan has been shown to be a bioactive substrate itself for dentin regeneration because it increased the expression of odontoblastic markers in DPCs in vitro (35) and induced dentinogenesis in vivo (36). According to Gupte and $\mathrm{Ma}$ (37), scaffold architecture, mechanical properties, and degradation rate should be evaluated to select the ideal material for this goal. The porous network seems to be essential, especially for cell homing therapy, to allow for surrounding tissue precursor and endothelial cell migration inside its structure, ultimately with new tissue deposition. We choose chitosan because a porous matrix can be easily fabricated, such as described in the present investigation, in which CHSC featured $35.1 \%$ porosity, allowing the cells to migrate inside the material structure. Therefore, chitosan-SIM scaffolds might be useful in direct pulp capping therapy because they may provide a biocompatible and bioactive matrix capable to modulate the dentinogenesis phenomenon by modulating the odontogenic potential of host cells.

To evaluate the potential of low-dose SIM scaffolds developed in the present laboratory study as a cell-free approach to dentin regeneration, we applied the biomaterials to an artificial pulp chamber model containing a 3D culture of DPCs. It was shown that CHSCSIM1.0 and CHSC-SIM0.5 induced the migration and adhesion of viable DPCs from 3D culture to the scaffold's surface, as observed by the Live/Dead assay. The cells in 3D culture exhibited an odontoblastic phenotype mediated by the SIM-loaded scaffolds because increased gene expression of ALP, Col1, DMP-1, and DSPP in the absence of osteogenic supplementation in the culture medium was detected. These expressions were more intensely mediated by CHSC-SIM1.0. The presence of these odontoblastic markers in the 3D culture and its correlation with the appearance of the calciumrich matrix on DPC/SIM scaffold constructs determines the potential for the CHSC-SIM1.0 biomaterial to act in the inducible chemotactic matrix, cell-mediated deposition, and regulation of the mineral phase, critical end points that are a hallmark feature of dentin regeneration. Therefore, this biomaterial may be considered an interesting alternative for the stimulation of regeneration of the pulp-dentin complex. 
However, considering the limitations of the data obtained in this in vitro study, following the recommendations for the development of new products and clinical protocols, we suggest that further investigations are needed to assess the potential of the CHSC-SIM1.0 biomaterial applied for in vivo models.

\section{Conclusion}

In conclusion, the incorporation of low-dose SIM into a macroporous chitosan scaffold indicates a chemotactic potential for DPCs, leading these cells to strongly express an odontoblastic phenotype and deposit high amounts of mineralized matrix.

\section{Acknowledgments}

Supported by the São Paulo Research Foundation-FAPESP (grants no. 2013/23520-0 and 2016/15674-5) and the National Council for Scientific and Technological Development-CNPq (grants no. 303599/2014-6 and 442336/2014-4).

The authors deny any conflicts of interest related to this study.

\section{Supplementary Material}

Supplementary material associated with this article can be found in the online version at www.jendodon.com (bttps://doi. org/10.1016/j.joen.2018.02.014)

\section{References}

1. Galler KM, Eidt A, Schmalz G. Cell-free approaches for dental pulp tissue engineer ing. J Endod 2014; 40:S41-5.

2. Piva E, Silva AF, Nör JE. Functionalized scaffolds to control dental pulp stem cell fate. J Endod 2014; $40:$ S33-40.

3. Schmalz G, Smith AJ. Pulp development, repair, and regeneration: challenges of the transition from traditional dentistry to biologically based therapies. J Endod 2014; 40:S2-5.

4. Rambhia KJ, Ma PX. Controlled drug release for tissue engineering. J Control Release 2015;219:119-28.

5. Soares DG, Rosseto HL, Scheffel DS, et al. Odontogenic differentiation potential of human dental pulp cells cultured on a calcium-aluminate enriched chitosancollagen scaffold. Clin Oral Investig 2017;21:2827-39.

6. Madihally SV, Matthew HW. Porous chitosan scaffolds for tissue engineering. Biomaterials 1999;20:1133-42.

7. Kim IY, Seo SJ, Moon HS, et al. Chitosan and its derivatives for tissue engineering applications. Biotechnol Adv 2008;26:1-21.

8. Liu YS, Ou ME, Liu H, et al. The effect of simvastatin on chemotactic capability of SDF- $1 \alpha$ and the promotion of bone regeneration. Biomaterials 2014;35:4489-98.

9. Gentile P, Nandagiri VK, Daly J, et al. Localised controlled release of simvastatin from porous chitosan-gelatin scaffolds engrafted with simvastatin loaded PLGAmicroparticles for bone tissue engineering application. Mater Sci Eng C Mater Biol Appl 2016;59:249-57.

10. Oryan A, Kamali A, Moshiri A. Potential mechanisms and applications of statins on osteogenesis: current modalities, conflicts and future directions. J Control Release 2015;215:12-24.

11. Kheirallah M, Almeshaly A. Simvastatin, dosage, and delivery system for supporting bone regeneration, an update review. J Oral Maxillofac Surg Med Pathol 2016;28: 206-9.

12. Okamoto Y, Sonoyama W, Ono M, et al. Simvastatin induces the odontogenic differentiation of human dental pulp stem cells in vitro and in vivo. J Endod 2009;35: $367-72$.

13. Min KS, Lee YM, Hong SO, Kim EC. Simvastatin promotes odontoblastic differentiation and expression of angiogenic factors via heme oxygenase- 1 in primary cultured human dental pulp cells. J Endod 2010;36:447-52.

14. Lee SY, Min KS, Choi GW, et al. Effects of simvastain and enamel matrix derivative on Portland cement with bismuth oxide-induced growth and odontoblastic differentiation in human dental pulp cells. J Endod 2012;38:405-10.
15. Zijah V, Salehi R, Aghazadeh M, et al. Towards optimization of odonto/osteogenic bioengineering:in vitro comparison of simvastatin, sodium fluoride, melanocytestimulating hormone. In Vitro Cell Dev Biol Anim 2017;53:502-12.

16. Basso FG, Soares D, Pansani TN, et al. Proliferation, migration, and expression of oral mucosal healing related genes by oral fibroblasts receiving low level laser therapy after inflammatory cytokines challenge. Lasers Surg Med 2016; 48:1006-14.

17. Soares DG, Brito CA, Tavares da Silva RH, et al. Cytocompatibility of HEMA-free resin-based luting cements according to application protocols on dentine surfaces. Int Endod J 2016;49:551-60.

18. Chen PY, Sun JS, Tsuang YH, et al. Simvastatin promotes osteoblast viability and differentiation via Ras/Smad/Erk/BMP-2 signaling pathway. Nutr Res 2010;30:191-9.

19. Kim IS, Jeong BC, Kim OS, et al. Lactone form 3-hydroxy-3-methylglutaryl-coenzyme A reductase inhibitors (statins) stimulate the osteoblastic differentiation of mouse periodontal ligament cells via the ERK pathway. J Periodontal Res 2011;46:204-13.

20. Karanxha L, Park SJ, Son WJ, et al. Combined effects of simvastatin and enamel matrix derivative on odontoblastic differentiation of human dental pulp cells. J Endod 2013;39:76-82.

21. Tai IC, Wang YH, Chen $\mathrm{CH}$, et al. Simvastatin enhances Rho/actin/cell rigidity pathway contributing to mesenchymal stem cells' osteogenic differentiation. Int J Nanomedicine 2015;21:5881-94.

22. Jia W, Zhao Y, Yang J, et al. Simvastatin promotes dental pulp stem cell induced coronal pulp regeneration in pulpotomized teeth. J Endod 2016;42:1049-54.

23. Han X, Yang N, Cui Y, et al. Simvastatin mobilizes bone marrow stromal cells migrating to injured areas and promotes functional recovery after spinal cord injury in the rat. Neurosci Lett 2012;521:136-41.

24. Nath SD, Linh NT, Sadiasa A, Lee BT. Encapsulation of simvastatin in PLGA microspheres loaded into hydrogel loaded BCP porous spongy scaffold as a controlled drug delivery system for bone tissue regeneration. J Biomater Appl 2014;28: 1151-63.

25. Nandagiri VK, Muttu C, Daly J, et al. Preparation and characterization of simvastatin loaded PLGA microparticles for tissue engineering applications. Curr Res Pharm Sci 2012;02:81-90.

26. Pestov A, Bratskaya S. Chitosan and its derivatives as highly efficient polymer ligands. Molecules 2016:21:330.

27. Tai IC, Fu YC, Wang CK, et al. Local delivery of controlled-release simvastatin/ PLGA/HAp microspheres enhances bone repair. Int J Nanomedicine 2013;8: 3895-904.

28. Bae MS, Yang DH, Lee JB, et al. Photo-cured hyaluronic acid-based hydrogels containing simvastatin as a bone tissue regeneration scaffold. Biomaterials 2011;32: $8161-71$.

29. Fukui T, Ii M, Shoji T, et al. Therapeutic effect of local administration of low-dose simvastatin-conjugated gelatin hydrogel for fracture healing. J Bone Miner Res 2012;27:1118-31.

30. Wadagaki R, Mizuno D, Yamawaki-Ogata A, et al. Osteogenic induction of bone marrow-derived stromal cells on simvastatin-releasing, biodegradable, nano-to microscale fiber scaffolds. Ann Biomed Eng 2011;39:1872-81.

31. Mendes Junior D, Domingues JA, Hausen MA, et al. Study of mesenchymal stem cells cultured on a poly(lactic-co-glycolic acid) scaffold containing simvastatin for bone healing. J Appl Biomater Funct Mater 2017;15:e133-41.

32. Yu WL, Sun TW, Qi C, et al. Enhanced osteogenesis and angiogenesis by mesoporous hydroxyapatite microspheres-derived simvastatin sustained release system for superior bone regeneration. Sci Rep 2017;7:44129.

33. Miyazawa A, Matsuno T, Asano K, et al. Controlled release of simvastatin from biodegradable hydrogels promotes odontoblastic differentiation. Dent Mater J 2015;34: 466-74.

34. Asl Aminabadi N, Maljaei E, Erfanparast L, et al. Simvastatin versus calcium hydroxide direct pulp capping of human primary molars: a randomized clinical trial. J Dent Res Dent Clin Dent Prospects 2013;7:8-14.

35. Amir LR, Suniarti DF, Utami S, Abbas B. Chitosan as a potential osteogenic factor compared with dexamethasone in cultured macaquedental pulp stromal cells. Cell Tissue Res 2014;358:407-15.

36. Li F, Liu X, Zhao S, et al. Porous chitosan bilayer membrane containing TGF- $\beta 1$ loaded microspheres for pulp capping and reparative dentin formation in a dog model. Dent Mater 2014;30:172-81.

37. Gupte MJ, Ma PX. Nanofibrous scaffolds for dental and craniofacial applications. J Dent Res 2012;91:227-34. 


\section{Supplementary Material}

\section{alamarBlue Assay [ $\boldsymbol{n}=\mathbf{6}]$}

Cells were incubated with alpha minimum essential medium ( $\alpha$-MEM) supplemented with alamarBlue dye (10:1; Thermo Fisher Scientific, Waltham, MA) at $37^{\circ} \mathrm{C}$ and $5 \% \mathrm{CO}_{2}$ for 4 hours, and fluorescence was read at $570 \mathrm{~nm}$ excitation and $585 \mathrm{~nm}$ emission (Synergy H1; BioTek, Winooski, VT). The mean value of the negative control group $(0 \mu \mathrm{mol} / \mathrm{L}$ SIM $)$ on day 1 was considered as $100 \%$ of cell viability.

\section{Live/Dead Assay [ $\boldsymbol{n}=\mathbf{2}]$}

The Live/Dead Viability/Cytotoxicity Kit (Invitrogen, Carlsbad, CA) was used. At each time point, the samples were washed once in phosphate-buffered saline and incubated for 45 minutes with $\alpha$-MEM (no fetal bovine serum) supplemented with $4 \mu \mathrm{mol} / \mathrm{L}$ ethyl homodimer-1 and $2 \mu \mathrm{mol} / \mathrm{L}$ calcein AM (CA). The material's surface and 0.5 -mm-thick transverse slices were analyzed by fluorescence microscopy (Leica DM 5500B; Nussloch GmbH, Nussloch, Germany).

\section{F-actin Staining [ $\boldsymbol{n}=\mathbf{2}]$}

The cell/scaffold constructs were washed in phosphate-buffered saline, fixed in 4\% paraformaldehyde (Sigma-Aldrich, St Louis, M0), permeabilized in $0.1 \%$ Triton X (Thermo Fisher Scientific), and incubated for 30 minutes with Alexa Fluor 488 Phalloidin probe (1:50; Life Technologies, Carlsbad, CA). Mounting medium with 4',6-diamidino-2-phenylindole (ProLong, Thermo Fisher Scientific) was used to stain the nuclei, and representative images were obtained at the material surface by fluorescence microscopy (Leica DM 5500B, Nussloch GmbH).

\section{Alkaline Phosphatase Activity $[\boldsymbol{n}=\mathbf{6}$ ]}

For thymolphthalein monophosphate substrate-based assay, cell lysis was performed with $0.1 \%$ sodium lauryl sulfate (Sigma-Aldrich), and alkaline phosphatase (ALP) activity was assessed with the Endpoint kit (Labtest, Lagoa Santa, MG, Brazil). For pNPP substrate-based assay, the scaffolds were subjected to manual disruption followed by incubation with the SensoLyteTM Alkaline Phosphatase Assay Kit (AnaSpec, Fremont, CA) and lysis buffer (Triton X-100, Thermo Fisher Scientific) for 10 minutes at $4^{\circ} \mathrm{C}$. The supernatant was collected by centrifugation $\left(10,000 \mathrm{~g}\right.$ at $4^{\circ} \mathrm{C}$ for 15 minutes $)$, and ALP activity was performed according to the manufacturer's instructions. Total protein quantification was performed by the Lowry/Folin-Ciocalteau method (5) for ALP activity normalization. Data were converted into percentage of ALP activity based on the group with no SIM supplementation (100\% ALP activity).

\section{Alizarin Red [ $\boldsymbol{n}=\mathbf{6}]$}

The cells or disc/scaffold set were fixed with $70 \%$ ethanol at $4^{\circ} \mathrm{C}$ and stained with alizarin red dye $(40 \mathrm{mmol} / \mathrm{L}, \mathrm{pH}=4.2$; SigmaAldrich). The samples were washed in deionized water (1 time for cells seeded on plates and 5 times for the cell/scaffold construct). Representative images from each group were obtained by light microscopy (Olympus BX51; Olympus, Miami, FL), and quantitative measurements were performed after matrix dissolution with $10 \%$ cetylpyridinium chloride (Sigma-Aldrich) followed by absorbance measurement at $570 \mathrm{~nm}$ (Synergy H1; BioTek, Winooski, VT). The group with no SIM supplementation was considered as $100 \%$ of mineralized nodule deposition.

\section{Wound Healing [ $\boldsymbol{n}=\mathbf{4}]$}

The cells were cultured in 24-well plates $(6 \times 104 /$ well $)$ for 24 hours. After this period, we created an in vitro wound by scratching the monolayer formed by the attached cells using a 5-mL pipette tip. Then, cells were incubated with medium supplemented or not with SIM for 24 hours. The cells were then fixed in $70 \%$ ethanol and stained with crystal violet (Sigma-Aldrich) for measurement of the wound area as described by Basso et al (16).

\section{Transwell Assay $[\boldsymbol{n}=\mathbf{4}]$}

The cells were seeded in 8- $\mu \mathrm{m}$ micropore transwell inserts (Corning, Corning, NY) followed by incubation at $37^{\circ} \mathrm{C}$ for 2 hours to allow for cell adhesion. Thereafter, the inserts were positioned on 24-well plates containing $1 \mathrm{~mL}$ complete $\alpha$-MEM supplemented or not with $0.1 \mu \mathrm{mol} / \mathrm{L}$ SIM followed by a 4 - or 24 -hour incubation time. The numbers of migrating cells were determined as previously described (12). For the scaffold assay, the inserts containing cultured cells were positioned in direct contact with the chitosan scaffold in $1 \mathrm{~mL}$ $\alpha$-MEM supplemented or not with $0.1 \mu \mathrm{mol} / \mathrm{L}$ SIM (5).

\section{Real-time Polymerase Chain Reaction Assay [ $[=4]$}

Total RNA was extracted with the RNAqueous-Micro Kit (Ambion, Austin, TX), and complementary DNA was reverse transcribed with the High Capacity cDNA Reverse Transcription Kit (Applied Biosystems, Foster City, CA) according to the recommended protocol. Real-time polymerase chain reaction quantification of messenger RNA was performed with Step0ne Plus equipment (Applied Biosystems) by the use of Taqman assays. Beta-actin was used as the constitutive gene. Data were calculated according to the $2^{\Delta \Delta \mathrm{CT}}$ equation, with the chitosan scaffold group for normalization. 